\title{
TRIANGULATION OF MANIFOLDS. II
}

\author{
BY R. LASHOF AND M. ROTHENBERG ${ }^{1}$
}

Communicated by William Browder, January 27, 1968

Main theorems. We will say that a manifold $M$ satisfies condition $\mathrm{S}$, if $\pi_{1}\left(M \times T^{k}\right)$ and $\pi_{1}\left(\partial M \times T^{k}\right)$ satisfy the conditions necessary for the splitting theorem to hold [6], [9].

THEOREM 4. Every closed topological manifold $M$, $\operatorname{dim} M \geqq 5$, $H^{4}\left(M ; Z_{2}\right)=0$, and satisfying condition $S$, admits a $P L$ manifold structure. ${ }^{2}$

Proof. By Theorem 3 and addendum to Theorem 2, the tangent bundle of $M^{n}$ lifts to a $\mathrm{PL}_{n}$-bundle. By the splitting theorem [6], [9], there is a PL-manifold $Q$ of the same tangential homotopy type as $M$. As in [5], proof of (c), we may immerse $M_{0}=M$-point in $Q$, to give $M_{0}$ a PL manifold structure. By Lees' Lemma [5], $M$ admits a PL manifold structure.

Remarks. 1. If we are given a lift of $\tau\left(M^{n}\right)$ to a $\mathrm{PL}_{n}$-bundle, we may drop the condition $H^{4}\left(M ; Z_{2}\right)=0$.

2. If we are given a bundle map of $\tau\left(M_{0}\right)$ into $\tau(Q), Q^{n}$ a PL manifold, we may drop condition $\mathrm{S}$ as well.

Theorem 5. Let $W^{n}, n \geqq 5$, be a topological h-cobordism between $P L$ manifolds. If $H^{3}\left(W ; Z_{2}\right)=0$, then $W$ admits a $P L$ manifold structure with the given structures on the boundary.

Proof. Say $\partial W=M_{1} \cup M_{2}$. Then we may define inclusions $\iota_{1}: M_{1}$ $\times I \rightarrow W, \iota_{2}: M_{2} \times I \rightarrow W$ using collar neighborhoods. (Take $\iota_{1} \mid M_{1} \times 0$ =identity and $\iota_{2} \mid M_{2} \times 1=$ identity.) Also we have retractions $r_{1}: W \rightarrow M_{1} \times I, \quad r_{2}: W \rightarrow M_{2} \times I$, where for example we may take $r_{2} \mid M_{2}: M_{2} \rightarrow M_{2} \times 1$ by the identity, $r_{2} \mid M_{1}: M_{1} \rightarrow M_{2} \times 0$ by a homotopy equivalence, and $r_{2} \iota_{2}=$ identity. Now these maps are covered by topological bundle maps; $\iota_{i}^{*}: \tau_{1} \oplus 1 \rightarrow \tau=\tau(W), \iota_{2}^{*}: \tau_{2} \oplus 1 \rightarrow \tau$, and $r_{2^{*}}: \tau \rightarrow \tau_{2} \oplus 1$ so that $r_{2^{*} l_{2}}=$ identity (since $M_{2} \times I$ is a deformation

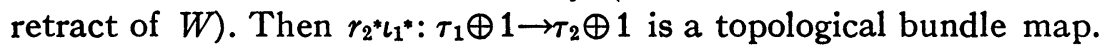

1 Partially supported by an NSF Grant.

2 As first shown by Kirby and Siebenmann (by other methods), condition $S$ may be eliminated. We can do this by applying Theorem 7 below to the normal disk bundle of a compact manifold $M$ (condition 3 is unnecessary since the tangent bundle is trivial) to obtain their result that $M$ is the homotopy type of a finite complex. The splitting theorem then holds with no condition on the fundamental group [9]. 
Now assume $r_{2}{ }^{*} \iota_{1}{ }^{*}$ is isotopic to a PL bundle map. Enlarge $W$ to the open $W^{\prime}=M_{1} \times(-1,0] \cup W \cup M_{2} \times[1,2)$. Then using Lees' Theorem we may immerse a neighborhood of $W$ in $M_{2} \times R$ by the bundle map $r_{2^{*}}: \tau \rightarrow r_{2} \oplus 1$. Since $r_{2^{*}} \iota_{2}^{*}=$ identity and $r_{2^{*}} \iota_{1}{ }^{*}$ is isotopic to a PL bundle map, we may assume the immersion $\phi$ satisfies $\phi_{2}: M_{2} \times[0,1]$ $\rightarrow M_{2} \times R$ is the inclusion, and $\phi \iota_{1}: M_{1} \times[0,1] \rightarrow M_{2} \times R$ is a PL immersion. Thus the immersion defines a PL structure on $W$, which agrees with the given structures on the boundary.

To show $r_{2}{ }^{*} \iota_{2}{ }^{*}$ is isotopic to a PL bundle map under the hypothesis that $H^{3}\left(W, Z_{2}\right)=H^{3}\left(M_{1} ; Z_{2}\right)=0$; note that topological (PL) bundle maps over $r_{2} \iota_{2}$ are given by cross-sections of an associated bundle $E^{\mathrm{Top}}\left(E^{\mathrm{PL}}\right)$ over $M_{1}$ with fibre $\operatorname{Top}_{n}\left(\mathrm{PL}_{n}\right)$. The map $\mathrm{PL}_{n} \rightarrow \mathrm{Top}_{n}$ induces a map $\rho: E^{\mathrm{PL}} \rightarrow E^{\mathrm{Top}}$. The fibre of $\rho$ is homotopy equivalent to $\Omega\left(\operatorname{Top}_{n} / \mathrm{PL}_{n}\right)$, which has at most one nontrivial homotopy class in dimensions $\leqq n-2$. This is in dimension 2 , and at most of order 2 . Thus the only obstruction to lifting a cross-section of $E^{\text {Top }}$ to one of $E^{\mathrm{PL}}$ lies in $H^{3}\left(M_{1} ; Z_{2}\right)=H^{3}\left(W ; Z_{2}\right)$.

THEOREM 6. Let $M^{n}$ be a compact topological manifold with boundary $N^{n-1}$, with fundamental groups satisfying conditions $S$.

(a) If $H^{4}\left(M, Z_{2}\right)=H^{3}\left(N, Z_{2}\right)=0$, and $n \geqq 6, M$ admits a PL manifold structure.

(b) If $N$ already has a PL structure, $H^{4}\left(M, Z_{2}\right)=H^{3}\left(N, Z_{2}\right)=0$ and $n \geqq 5$, then $M$ admits a $P L$ manifold structure agreeing with the given one on the boundary.

Proof. (a) If $H^{4}\left(M ; Z_{2}\right)=0$, then $\tau(M)$ lifts to a $\mathrm{PL}_{n}$ bundle. This induces a lift of $\tau(N) \oplus 1$ to a $\mathrm{PL}_{n}$ bundle; which lifts in turn to a $\mathrm{PL}_{n-1}$ bundle $\alpha$ over $N$, since $\pi_{i}\left(\mathrm{PL}_{n}, \mathrm{PL}_{n-1}\right)=0$, for $i \leqq n-2$. The lift is unique, except on the top cell of $N$. Since $\pi_{n-1}\left(\mathrm{PL}_{n}, \mathrm{PL}_{n-1}\right)$ $\simeq \pi_{n-1}\left(\operatorname{Top}_{n}\right.$, Top $\left._{n-1}\right)$ (see proof of Theorem 3 of I), we may choose $\alpha$ to be a lift of $\tau(N)$. As in the proof of Theorem $1, N$ may be triangulated. This reduces case (a) to case (b).

(b) Take the double of $M$. It admits a PL structure by Theorem 4. Hence $W=M-N$ has a PL structure. By (9), $W$ is collared. $W$ $=\bar{W} \cup \mid \partial \bar{W} \times[0,1)$. Hence $M=\bar{W} \cup V$ where $V$ is an $h$-cobordism between $\partial \bar{W}$ and $\partial M$. By Theorem $2, V$ admits a PL structure which extends the one on $\partial V=\partial \bar{W} \cup N$. Q.E.D.

Instead of using the splitting theorem to construct a PL manifold of the same tangential homotopy type, one may use the surgery techniques of Browder, Novikov, and Wall [12]. As an example we get

THEOREM 7. Let $M^{n}$ be a compact connected manifold with boundary $N$, such that 
1. each component of $N$ except one, $N_{0}$, has a $P L$ structure.

2. $\pi_{1}\left(N_{0}\right) \rightarrow \pi_{1}(M)$ is an isomorphism.

3. $H^{3}\left(N ; Z_{2}\right)=H^{4}\left(M ; Z_{2}\right)=0$.

Then $M$ admits a PL structure which extends the given ones on the boundary components.

\section{BIBLIOGRAPHY}

1. A. Haefliger and V. Poenaru, La classification des immersions cocombinatoires, Inst. Hautes Etudes Sci. Publ. Math. No. 23, 1964, pp. 75-91.

2. W. C. Hsiang and J. L. Shaneson, Fake tori and the annulus conjecture, Mimeographed Notes, Yale University, New Haven, Conn., 1968.

3. R. C. Kirby, Stable homeomorphisms, Mimeographed Notes, Institute for Advanced Study, Princeton, N. J., 1968.

4. J. Lees, Immersions and surgeries on topological manifolds, Mimeographed Notes, Rice University, Houston, Tex., 1968.

5. R. Lashof, Lees' immersion theorem and the triangulation of manifolds, Mimeographed Notes, University of Chicago, Chicago, Ill., 1968.

6. R. Lashof and M. Rothenberg, Hauptvermutung for manifolds, Conference on the Topology of Manifolds, Complementary Series in Mathematics, Prindle, Weber and Schmidt, Boston, Mass., 1968, pp. 81-105.

7. J. Shaneson, Embeddings with codimension two of spheres in spheres, Bull. Amer. Math. Soc. 74 (1968), 972-974.

8. C. T. C. Wall, On homotopy tori and the annulus theorem (to appear).

9. L. Siebenmann, The obstruction to finding a boundary for an open manifold of dimension greater than five, Thesis, Princeton University, Princeton, N. J., 1965.

10. M. Hirsch, On tubular neighborhoods, Conference on the Topology of Manifolds, Prindle, Weber and Schmidt, Boston, Mass., 1968, pp. 63-80.

11. F. T. Farrell, Ph.D. Thesis, Yale University, New Haven, Conn., 1967.

12. C. T. C. Wall, Surgery on compact manifolds, Mimeographed Notes, Liverpool, 1967.

University of Chicago, Chicago, Illinois 60637 